\title{
A UTILIZAÇÃO DE MODELOS BIM NA GESTÃO DE RESÍDUOS DE CONSTRUÇÃO E DEMOLIÇÃO ${ }^{1}$
}

\author{
THE USE OF BIM MODELS IN CONSTRUCTION AND DEMOLITION \\ WASTE MANAGEMENT
}

\author{
Henrique José Silva de Carvalho \\ Universidade Federal do Paraná (UFPR) \\ eng.henriquecarvalho@gmail.com \\ Sérgio Scheer \\ Universidade Federal do Paraná (UFPR) \\ scheer@ufpr.br
}

\begin{abstract}
Resumo
A produção significativa de resíduos é um grave problema enfrentado nos centros urbanos. O crescimento populacional, o desenvolvimento econômico e a utilização de tecnologias inadequadas, aumentam a quantidade diária de resíduos enviados aos aterros. O presente trabalho apresenta o uso de BIM (Building Information Modeling) como ferramenta para auxiliar no processo de gestão de resíduos. Diante do cenário do país, caracterizado pelo grande número de obras e criação de leis como a Política Nacional de Resíduos Sólidos (Lei 12.305/10) é fundamental o estabelecimento de metas de geração e quantificação mais precisa dos resíduos. 0 uso de BIM rompe as barreiras da simples modelagem 3D. A parametrização permite que durante a elaboração do projeto, indicadores de sustentabilidade sejam simulados antes da execução das obras. A partir das questões levantadas, o trabalho apresenta estudos e pesquisas realizadas onde o BIM aparece como alternativa para a melhoria do processo de gestão de resíduos. Os autores pesquisados apresentam a correlação existente entre projetos concebidos com objetivo de menor geração e sistemas criados para a quantificação de RCD em modelos virtuais. Dessa forma é possível verificar que o BIM contribuiu para a gestão de RCD, possibilitando decisões que serão refletidas nos canteiros de obras.
\end{abstract}

Palavras-chave: Gestão de resíduos. BIM. Sustentabilidade.

\begin{abstract}
The significant waste production is a serious problem faced in urban centers. Population growth, economic development and the use of inappropriate technologies, increase the daily amount of waste sent to landfills. This paper presents the use of BIM (Building Information Modeling) as a tool to assist in the waste management process. On the scenario of the country, characterized by the large number of works and creation of laws such as the National Policy on Solid Waste (Law 12,305 / 10) it is crucial to establish generation targets and more precise quantification of the waste. The use of BIM breaks the barriers of simple 3D modeling. During the project design phase the parameterization allows to simulate sustainability indicators before the execution of works. From the issues raised, this work carried out studies and research where the BIM appears as an alternative to improve the waste management process. The presented literature review shows the correlation between projects designed with the objective to generate less waste and systems developed for the construction and demolition waste (RCD) quantification using virtual models. This way you can check the BIM contributed to the RCD management, enabling decision-making be reflected in the construction sites.
\end{abstract}

Keywords: Waste Management. BIM. Sustainability.

\footnotetext{
1 CARVALHO, H.; SCHEER, S. A utilização de modelos BIM na gestão de resíduos de construção e demolição. In: ENCONTRO BRASILEIRO DE TECNOLOGIA DE INFORMAÇÃO E COMUNICAÇÃO NA CONSTRUÇÃO, 7 ., 2015, Recife. Anais... Porto Alegre: ANTAC, 2015.
} 


\section{的}

\section{TIC2015}

\section{INTRODUÇÃO}

Desde 2011, a indústria da construção civil vem alcançando índices expressivos de crescimento. No período de 2011 a 2012 o PIB setorial teve um crescimento de 1,5\%, acima da média do PIB nacional, com $0,9 \%$. O cenário apresentado, apesar de ser de grande expressão econômica, se mostra negativo no que diz respeito à quantidade de energia e recursos naturais que a construção civil utiliza (DIEESE, 2013).

Na construção civil o consumo de recursos naturais está ligado a fatores como: (i) taxa de resíduos gerados; (ii) ciclo de vida das estruturas construídas; (iii) atividades de manutenção e correção de falhas construtivas; (iv) perdas incorporadas nos edifícios; (v) tecnologia construtiva utilizada (JOHN, 2000).

Os impactos ocasionados pelas atividades da construção civil são constantemente discutidos pela literatura, sendo destaque a grande quantidade de resíduos gerados pelo setor. Observa-se que grande parte dos estudos realizados apresenta os processos de reciclagem dos resíduos, seja nos canteiros ou em áreas específicas a este fim, como uma possível solução à problemática, contudo, existe ainda uma pouca utilização de materiais reciclados pela indústria da construção civil, principalmente os fabricados com resíduos Classe A.

A Política Nacional de Resíduos Sólidos (PNRS) - Lei 12.305/10 (BRASIL, 2010), determina que as empresas de construção civil, geradoras de resíduos de construção e demolição (RCD) devem estabelecer e operacionalizar planos de gerenciamento que garantam a coleta seletiva, recuperação e reciclagem, tratamento e destinação final dos mesmos.

De acordo com Pinto (1999), no Brasil não existem levantamentos precisos para relacionar a exata produção de resíduos dos diversos agentes que atuam na construção, dificultando a análise do volume gerado pela construção civil nas áreas urbanas.

A dificuldade de uma quantificação mais precisa dos resíduos dificulta um processo mais efetivo de gestão. Jalali (2007) aponta que a quantificação dos resíduos é fundamental para uma gestão ambientalmente e economicamente eficaz.

O uso de BIM (Building Information Modeling) na gestão de resíduos de construção e demolição já é tema de pesquisa de autores que apresentam a modelagem como uma solução para a quantificação destes resíduos, melhorando processos e incorporando aos planos de gestão informações que podem ser acessadas durante todo o ciclo de vida do empreendimento.

Este artigo aborda a utilização da modelagem paramétrica nos processos de gestão de resíduos, através da discussão dos resultados de pesquisas realizadas, que apontaram o uso do BIM como um grande avanço no processo de quantificação.

\section{GESTÃO DE RESÍDUOS DE CONSTRUÇÃO E DEMOLIÇÃO}

Conforme apresentado por Bernstein (1996), a Civil Engineering Research Foundation CERF, entidade dedicada a promover a modernização da construção civil dos Estados Unidos, realizou uma pesquisa com cerca de 1.500 construtores, projetistas e pesquisadores em todo o mundo com o objetivo de apontar as tendências consideradas fundamentais para o futuro do setor (Figura 1). 


\section{1}

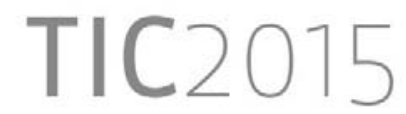

Figura 1: Grau de impacto de diferentes tendências nas atividades da construção civil

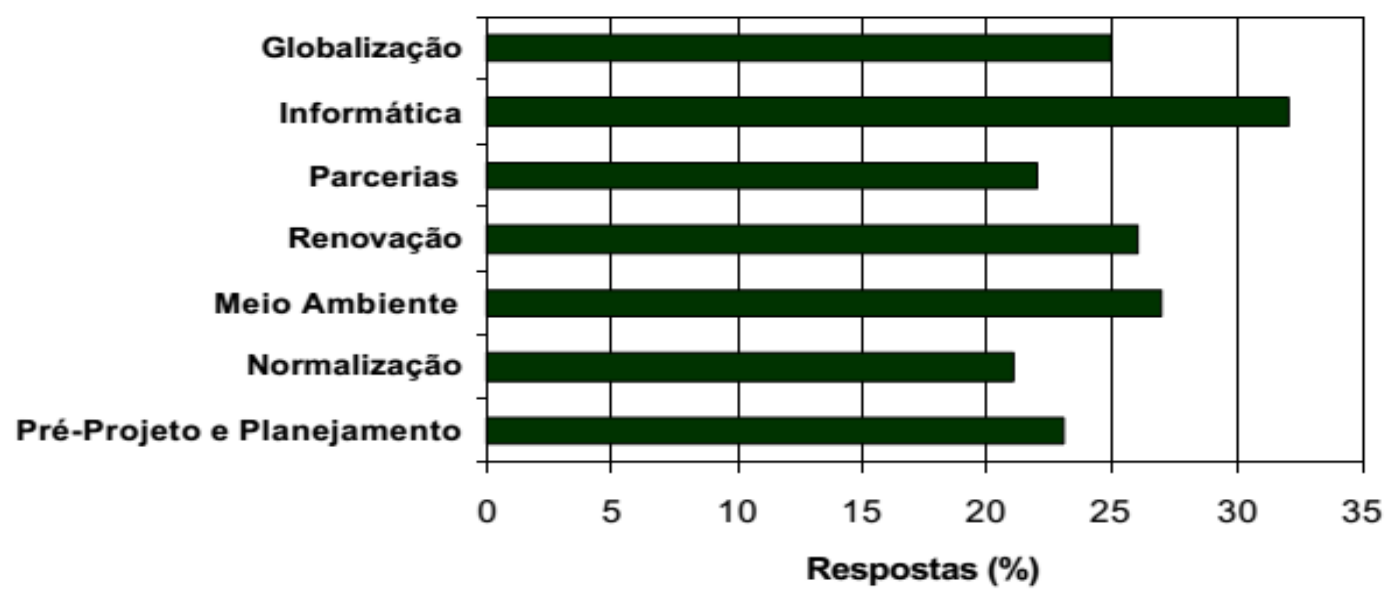

Fonte: Bernstein, 1996

Conforme Toledo et al. (2000), muitas mudanças e inovações tecnológicas, sendo algumas destas estratégias competitivas entre empresas da indústria da construção civil, estão sendo implementadas, contudo, algumas incertezas quanto à implantação de determinada tecnologia são obstáculos para o avanço das inovações. Os autores discutem que muitas empresas só adotam determinada tecnologia após esta ter sido testada e amplamente divulgada, apontando ainda que a necessidade de projetos multidisciplinares e a dependência do desenvolvimento de novos materiais e equipamentos travam ainda mais as inovações na construção civil.

Segundo John e Agopyan (2011), a indústria da construção civil demorou a discutir e enfrentar os problemas dos impactos ambientais, sendo que até a metade da década de 1990 não tinha sido colocada como uma indústria com problemas relacionados ao meio ambiente.

Ao se confrontar os dados da pesquisa apresentada por Bernstein (1996) e as afirmativas de Toledo et al. (2000); John e Agopyan (2011) é observado que os itens mais votados na pesquisa, informática (tecnologia) e meio ambiente, não avançaram tanto no Brasil segundo à tendência encontrada.

Os resíduos gerados pelo setor da construção civil têm promovido uma série de discussões que culminam em políticas públicas. O desperdício de materiais na forma de resíduos (comumente denominado entulho pela construção civil) é o mesmo que minimizar recursos naturais, argumento que insere a indústria da construção no centro de questionamentos em busca de sua dimensão sustentável (SOUZA et al., 2004).

Tessaro et al. (2012) discute que a produção de entulho é diretamente proporcional ao desenvolvimento socioeconômico, apontando que o crescimento dos grandes centros e os processos de urbanização são fatores que afetam diretamente a geração de resíduos.

No Brasil, a Lei Federal $n^{\circ}$ 10.257, promulgada em 2001, conhecida como Estatuto da Cidade, regulamentou importantes diretrizes na busca pelo desenvolvimento sustentável nos centros urbanos. De acordo a legislação o ambiente natural e o construído devem ser preservados, sendo distribuído entre eles o ônus da urbanização. A participação dos municípios é fundamental no processo por meios de políticas alinhadas ao seu Plano Diretor, dentre as quais se destaca a gestão dos resíduos urbanos e, por conseguinte os resíduos de construção e demolição (KARPINSKI, 2008).

A preocupação sobre a questão dos resíduos de construção civil sempre foi abordada e sinalizada por autores e pelo poder da legislação. Os processos de licenciamento de obras 
sempre exigiram, dentro da série de documentos necessários, um Plano de Gerenciamento de Resíduos - PGRS quase sempre elaborado por empresas de consultoria que atestam a responsabilidade do empreendimento, quanto ao descarte correto do material que sairá do canteiro. Um fato discutível nos PGRS elaborados é a estimativa de geração de resíduos, muitas vezes fundamentada em dados da literatura e em indicadores do mercado, não havendo desta forma uma correlação com a produtividade e perda de material da empresa contratante.

Como forma de reduzir o impacto ambiental causado pelos resíduos de construção a reciclagem aparenta ser uma excelente solução. Entretanto, a busca pela redução do desperdício ao máximo e por consequência a diminuição do consumo de recursos naturais é preferível dentro de uma visão mais sustentável (MOREIRA, 2010).

Kilbert apud John (2001) aborda que de forma geral, a busca pela redução dos impactos ocasionados pela indústria da construção civil é uma atividade complexa, onde várias ações precisar ocorrer de forma alinhada e simultânea. O autor aponta que o setor precisa:

- Minimizar o consumo de recursos;

- Maximizar a reutilização de recursos;

- Utilizar recursos renováveis e/ou recicláveis;

- Priorizar um ambiente saudável e não tóxico;

- Proteger o meio ambiente;

- Buscar a qualidade na criação do ambiente construído.

A busca pela sustentabilidade na indústria da construção civil, ainda que seja uma tendência como observada na pesquisa de Bernstein (1996), é um desafio constante diante do cenário econômico do país e dos processos tradicionalistas do setor. A inovação vem sendo um propósito de poucos mesmo que se observe a necessidade da consolidação mecanismos de desenvolvimento focados na racionalidade ambiental. Dentro dessa visão, à preservação de recursos, o reaproveitamento e a correta destinação dos resíduos, são premissas a serem incorporadas pela construção civil.

\section{BUILDING INFORMATION MODELING}

Contextualizado por Menezes (2011), a Modelagem da Informação na Construção (ou como preconizado pela ABNT (2009), Modelagem da Informação da Construção), tradução para o termo Building Information Modeling (BIM) é uma filosofia e ao mesmo tempo um processo de trabalho onde arquitetos, engenheiros e construtores (AEC), planejam e criam modelos virtuais integrados a um banco de dados com informações como: subsídios para orçamentação, previsão das fases de construção, características de materiais, entre outras atividades.

Construir o empreendimento virtualmente no computador antes da construção real é a proposta e um grande ganho oferecido pelo uso de BIM. Esse processo é um arranjo virtual cooperativo que reúne profissionais de diversas disciplinas, garantindo que o conhecimento agregado de cada um deles seja integrado em uma única fonte de dados, o modelo paramétrico da edificação (EASTMAN et al., 2014).

Também entendida como tecnologia, a Modelagem da Informação da Construção está sendo absorvida pela indústria da construção civil por oferecer o recurso de compartilhar informações sobre uma edificação, estruturando uma base confiável de apoio a decisões e melhorias dos processos no ciclo de vida do empreendimento. O banco de dados 
configurado junto ao modelo virtual não representa apenas características gráficas, oferecendo informações funcionais dessa edificação (NBIMS, 2007).

Souza et al. (2009) descreve que o uso de BIM propicia um aumento da quantidade de informações disponíveis nos projetos realizados. $O$ autor apresenta que a qualidade e precisão dessas informações, oferecem ao usuário da tecnologia acesso aos dados diante do modelo virtual da edificação. A parametrização dos objetos auxilia na tomada de decisão após simulações realizadas diretamente no modelo paramétrico. Com o modelo da edificação contendo informações gráficas e não gráficas as simulações passam a ser uma nova alternativa para a obtenção e antecipação de respostas.

Muitas são as possibilidades da utilização de BIM. A parametrização e a associação desta a um banco de dados com informações precisas, acessíveis e editáveis, permite que o usuário da ferramenta tenha o controle das suas decisões e promova discussões para ajustes e melhorias seja no projeto, na construção e/ou operacionalização deste.

A possibilidade da parametrização de indicadores com a modelagem da informação, fornece uma visão ampla dessa tecnologia e suas ferramentas, deixando de lado o uso da modelagem meramente para criação de maquetes virtuais, sugerindo a amplitude da usabilidade da ferramenta como apresentado na Figura 2, adaptada de Liu et al. (2011).

Figura 2: Usabilidade das ferramentas BIM

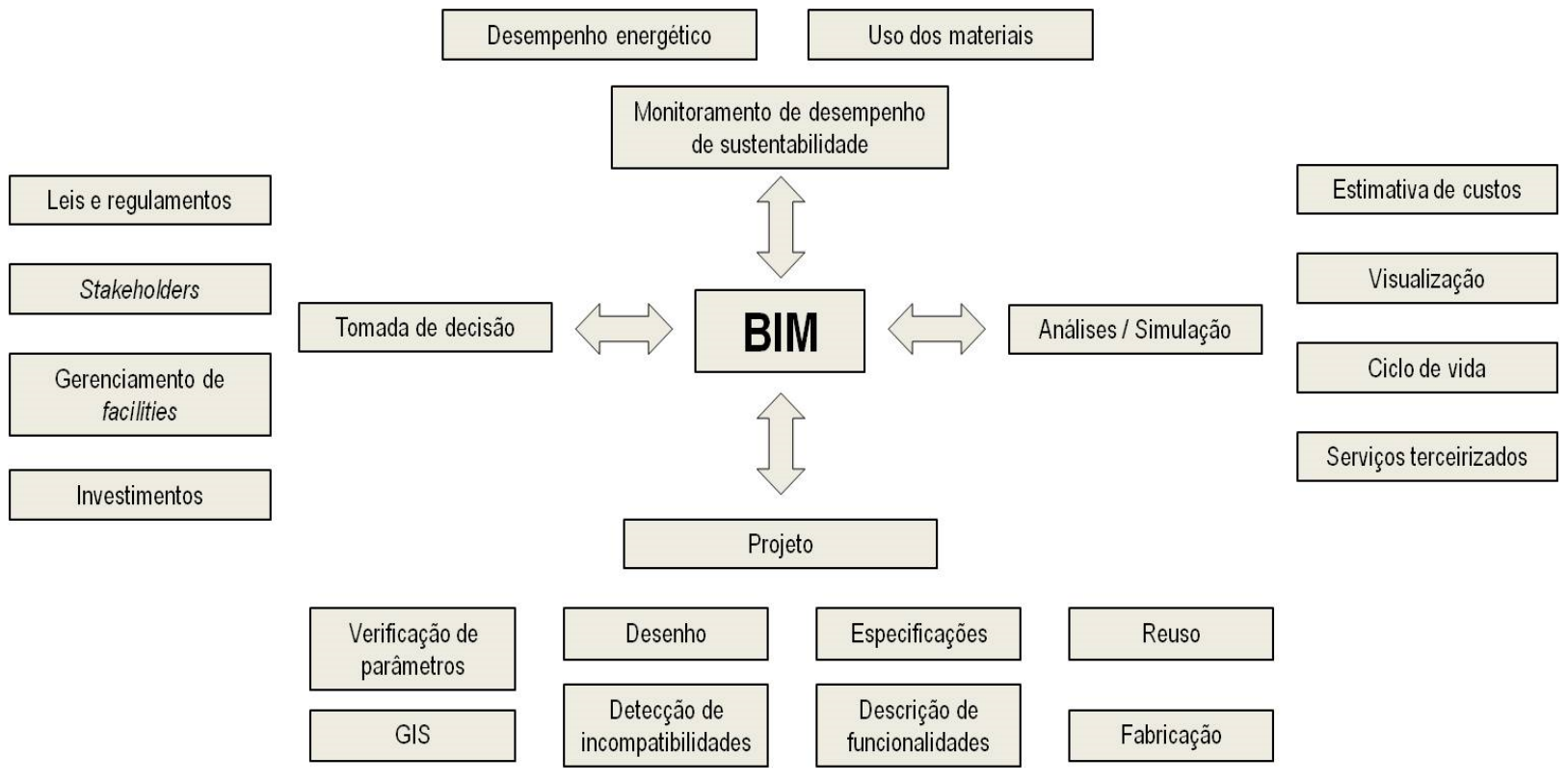

Fonte: Adaptado e traduzido de Liu et al. (2011)

Azevedo (2009) apresenta que são algumas das vantagens no uso de modelos BIM:

- Melhor gestão de materiais, reduzindo o desperdício de no canteiro de obras;

- Melhor gestão de programação de obras através da eliminação de erros no levantamento de quantitativos;

- Cronogramas de obras mais precisos. 
O BIM é uma tecnologia capaz de reduzir o desperdício prevendo os possíveis erros da etapa de construção desde a fase de projeto. A capacidade de detectar interferências na fase inicial dos projetos reflete positivamente durante a etapa construtiva. A gestão da informação associada ao uso das ferramentas BIM, permite incorporar a modelagem, comunicação, colaboração e integração de requisitos de concepção, dados e ações que farão parte de todo o ciclo de vida da edificação, como colocado por Cheng e Ma (2013).

\subsection{O BIM na gestão de resíduos de construção e demolição}

Não foi possível identificar trabalhos sobre o uso de BIM em projetos visando à quantificação dos resíduos a serem gerados e a proposição de soluções para a gestão ainda é desconhecida no Brasil. Entretanto, alguns pesquisadores estrangeiros propõem uma série de reflexões ao uso e aplicação prática da ferramenta.

Baldwin et al. (2007) investigaram a utilização de BIM para avaliar as opções de redução de resíduos de construção em edifícios residenciais. As pesquisas dos autores concluíram que é uma excelente alternativa para o desenvolvimento da análise da geração de resíduos de construção e as implicações destes nas decisões do projeto.

O trabalho dos autores Liu et al. (2011) identificam que na maioria dos projetos a usabilidade das ferramentas BIM para critérios de sustentabilidade é correlacionado a simulações de desempenho sustentável, principalmente para questões voltadas à energia. Dentro do contexto da pesquisa os autores identificam que a modelagem da informação pode ser usada como um sistema de comunicação colaborativo com potencial para auxiliar projetistas e stakeholders a atingirem de forma mais precisa a minimização de resíduos de construção em seus projetos.

Rajendran e Gomez (2012) discutem que muitas publicações discutem a gestão de resíduos, entretanto, a maioria destas apresentam formas de melhorar o local da gestão e atividades de reciclagem, havendo poucas tentativas de proposições de melhoria de projetos com o uso de BIM visando uma efetiva minimização de resíduos. Os autores contextualizam o conceito do designing-out-waste, como uma solução para a melhoria dos processos de gestão de resíduos.

Dentro do trabalho de Rajendran e Gomez (2012), é apresentada uma pesquisa realizada pela Waste \& Resources Action Programme (WRAP) apontando a importante contribuição que os projetistas podem fazer para a redução de resíduos através do projeto. Como resultado do trabalho a WRAP definiu cinco princípios que os projetistas devem utilizar visando à redução de resíduos durante a concepção de produtos, sendo eles:

- Projeto para o reuso e reciclagem;

- Projeto para fora da construção;

- Projeto para otimização de materiais;

- Projeto para aquisição eficiente de produtos;

- Projeto para flexibilidade na demolição.

Ainda de acordo com estes autores, algumas metas de sustentabilidade devem ser cumpridas pelos projetistas com o objetivo de desenvolverem edificações mais sustentáveis (Figura 3). 
Figura 3: Eficiência dos recursos materiais como parte da sustentabilidade nas construções

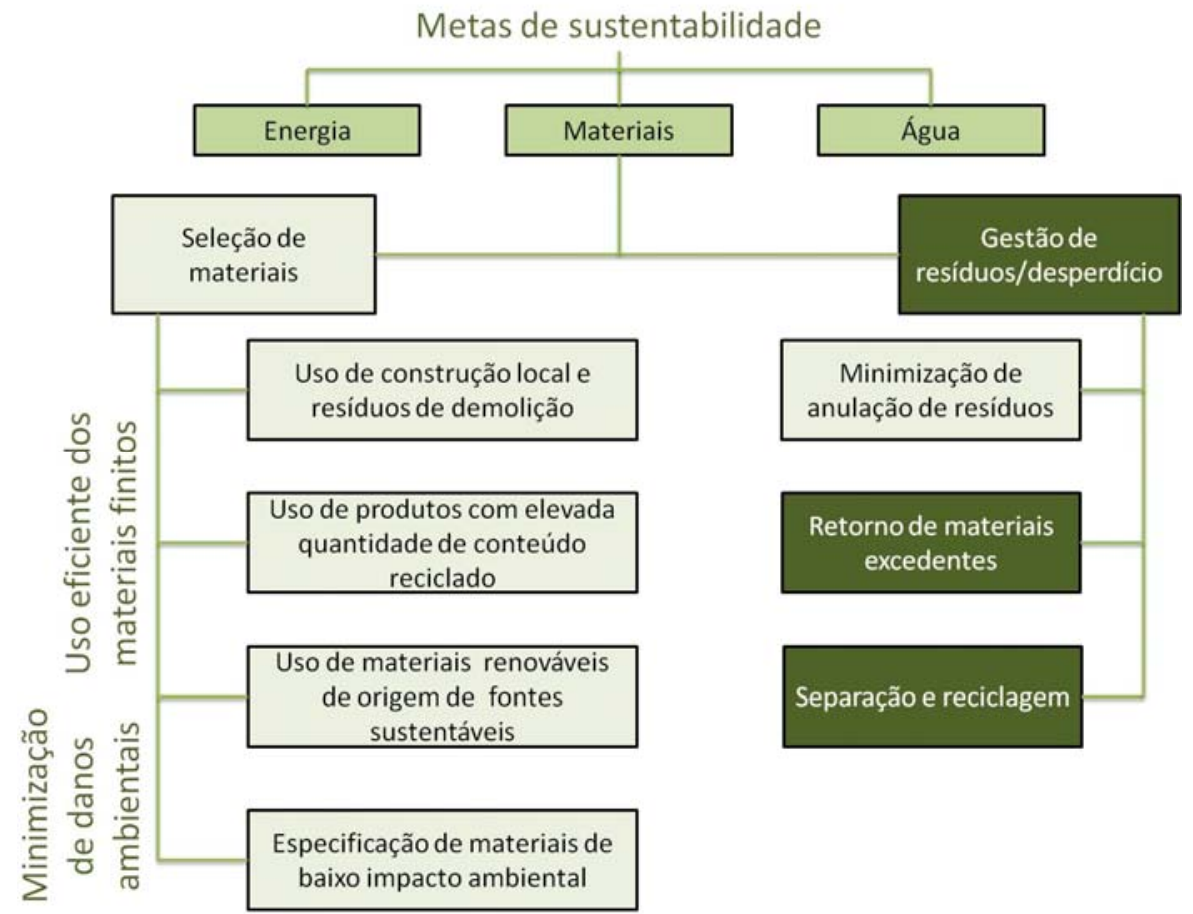

Fonte: Traduzido e Adaptado de WRAP (2010)

A análise dos resultados da pesquisa aponta que somente as melhorias em aspectos do projeto seriam suficientes para a minimização de resíduos. Entretanto, existem outras áreas que poderiam contribuir como processos de construção, gestão de materiais e planejamento. A utilização de BIM na fase de projetos ofereceria muitos dados relevantes para a gestão dos resíduos de forma efetiva nas demais etapas.

Cheng e Ma (2013) destacam as múltiplas informações que podem ser integradas ao modelo virtual parametrizado em BIM. No sistema de informações desenvolvido pelos autores, a parametrização de dados como o volume dos materiais de construção, permite uma estimativa dos resíduos a serem gerados.

Existem vários métodos para a quantificação de resíduos de construção e demolição, conforme apresentados pelos autores Cheng e Ma (2013):

- Estimativa baseada na área construída;

- Estimativa baseada nos componentes de um edifício;

- Estimativa baseada na entrada e saída de estoques de materiais;

- Estimativa baseada em dados de quantificação de outras construções;

- Estimativa de acordo com as formas físicas do layout.

A parametrização em software BIM permite quantificar a geração de resíduos já na elaboração do projeto. Para tanto é preciso definir um método de quantificação e parametrizá-lo na ferramenta que irá realizar a simulação.

Ainda segundo Cheng e Ma (2013), algumas aplicações mais específicas como o detalhamento de estruturas de aço, análise de energia e estimativas de custo, estão sendo 
cada vez mais desenvolvidas com o uso de BIM. Estas aplicações sempre são focadas na fase de projeto, não havendo nenhuma relacionada à fase de demolição. Através de suas pesquisas, os autores desenvolvem um sistema para estimar a geração de resíduos de construção e demolição, utilizando informações de volume como referência técnica para determinar o quantitativo. O sistema desenvolvido em software BIM é parametrizado de forma a fornecer as seguintes funções:

- Informações de volume detalhada para diferentes classes de resíduos;

- Informações sobre o volume de cada tipo de material;

- Volumes totais de resíduos inertes e não inertes;

- Estimativa de descarte de resíduos

- Estimativa de veículos necessários para logística dos resíduos.

\section{CONCLUSÃO}

Diversos autores apresentam a indústria da construção civil como um setor da economia que responde por uma parcela considerável no processo de degradação do meio ambiente, seja pela necessidade de recursos naturais para a fabricação dos seus insumos ou pela alta geração de resíduos inerente às técnicas construtivas utilizadas.

O uso da Modelagem da Informação da Construção (Building Information Modeling) vem sendo cada dia mais absorvida pelo setor. A criação e utilização de modelos paramétricos possibilita que simulações sejam realizadas antes da etapa de construção nos canteiros de obra. Diante desse contexto é muito aderente a utilização de ferramentas BIM no processo de quantificação dos resíduos gerados.

Este artigo abordou a utilização de modelagem paramétrica e BIM em apoio a atividades de gestão de resíduos, traçando um potencial panorama de aplicação e preparando o desenvolvimento de diretrizes para o desenvolvimento de aplicações de modelagem paramétrica na gestão de resíduos na Construção Civil. O levantamento realizado permite indicar que o desenvolvimento de aplicações baseadas em BIM podem ser instrumentos efetivos de apoio gerencial e estratégico, resultando em incremento de uso de materiais reciclados advindos das atividades de construção.

A pouca utilização de softwares BIM com o objetivo de mensuração de resíduos gerados ou mesmo para a gestão efetiva dos resíduos dos canteiros é muito evidente no país. A experiência de autores internacionais nesse quesito colabora para que dentro em breve algumas práticas de projetos com foco no design-out-waste sejam elaboradas no Brasil, com o planejamento correto de reaproveitamento e descarte de alguns materiais de forma minimizada e ambientalmente responsável.

É possível verificar que o uso de BIM favorece a melhoria da gestão de resíduos nos canteiros. O modelo desenvolvido durante o projeto pode oferecer dados e informações que possibilitam uma tomada de decisão mais efetiva na etapa construtiva.

\section{REFERÊNCIAS}

AZEVEDO, O. J. M. Metodologia BIM - Building Information Modeling na direção técnica de obras. Dissertação (Mestrado em Engenharia Civil, Reabilitação, Sustentabilidade e Materiais de Construção). Escola de Engenharia, Universidade do Minho, 2009. 
BALDWIN, A. et al. Reducing construction waste by decisions within the design process. In: CIB World Building Congress 2007. XVII, 2007, Cape Town, África do Sul, Anais..., Cape Town, 2007.

BERNSTEIN, $\mathrm{H}$. Bridging the globe: creating an intemational climate and challenges of sustainable design and construction. Industry and environment. Paris, v. 29, n.2, p. 19-21, 1996.

BRASIL. Lei $n^{\circ}$ 12.305, de 2 de agosto de 2010. Política Nacional de Resíduos Sólidos. Portal da Legislação Governo Federal. Disponível em: <https://www.planalto.gov.br/ccivil_03/_ato2007-2010/2010/lei//12305.htm>. Acesso em 02 abr. 2015.

CHENG, J. C. P.; MA, L. Y. H. A BIM-based system for demolition and renovation waste estimation and planning. Waste Management v. 33, n. 6, p. 1539-1551, 2013.

DIEESE - Departamento Intersindical de Estatística e Estudos Sócios Econômicos. Estudo Setorial da Construção Civil 2012, N65, Maio, 2013. Disponível em < https://www.dieese.org.br/estudosetorial/2012/estPesq65setorialConstrucaoCivil2012.pdf>. Acesso em 25 mar. 2015.

EASTMAN, C. et al. Manual de BIM. Um guia de modelagem da informação da construção para arquitetos, engenheiros, gerentes, construtores e incorporadores. Editora Bookman, Porto Alegre, 2014.

JALALI, S. Quantification of construction waste amount. In: International Technical Conference of Waste, 6, 2007, Viseu, Portugal, Anais..., Viseu, 2007.

JOHN, V. M. Reciclagem de resíduos na construção civil: contribuição para metodologia de pesquisa e desenvolvimento. Tese (Livre Docência), Escola Politécnica, Universidade de São Paulo, São Paulo, 2000.

JOHN, V. M. Aproveitamento de resíduos sólidos como materiais de construção. In: Reciclagem de Entulho para Produção de Materiais de Construção: Projeto Entulho Bom, EDUFBA (Editora da Universidade Federal da Bahia), Salvador, 2001.

JOHN, V. M.; AGOPYAN, V. O desafio da sustentabilidade na construção civil. Editora Blucher. Volume 5. São Paulo, 2011.

KARPINSKI, L. A. et al. Gestão de resíduos da construção civil: uma abordagem prática no município de Passo Fundo-RS. Estudos tecnológicos em engenharia, v. 4, n. 2, p. 69-87, São Leopoldo, 2008.

LIU, Z. et al. The potential use of BIM to aid construction waste minimisation. In: International Conference CIB World Building Congress 2011, Sophia Antipolis, France, Anais..., Sophia Antipolis, 2011.

MENEZES, G. L. B. B. Breve histórico de implantação da plataforma BIM. Caderno de Arquitetura e Urbanismo, Departamento de Arquitetura e Urbanismo da PUC Minas. v. 18, n. 22, p-153-171, Belo Horizonte 2011.

MOREIRA, L. H. H. Avaliação da influência da origem e do tratamento dos agregados reciclados de resíduos de construção e demolição no desempenho 


\section{1}

mecânico do concreto estrutural. Dissertação (Mestrado em Engenharia), Escola Politécnica, Universidade de São Paulo, São Paulo, 2010.

NBIMS - National Building Information Modeling Standard - Version 1 - Part 1: Overview, Principles, and Methodologies. Washington, 2007.

PINTO, T. P. Metodologia para a gestão diferenciada de resíduos sólidos da construção urbana. Tese (Doutorado em Engenharia) - Escola Politécnica, Universidade de São Paulo, São Paulo. 1999.

RAJENDRAN, P.; GOMEZ, C. P. Implementing BIM for waste minimisation in the construction industry: a literature review. In: International Conference on Management 2012, II, Langkawi Kedah, Malaysia, Anais..., Langkawi Kedah, 2012.

SOUZA, U. E. L. de et al. Diagnóstico e combate à geração de resíduos na produção de obras de construção de edifícios: uma abordagem progressiva. Ambiente Construído, v. 4, n. 4, p. 33-46, Porto Alegre, 2004.

SOUZA, L. L. A. et al. Impactos do uso do BIM em escritórios de arquitetura: Oportunidades no mercado imobiliário. Gestão \& Tecnologia de Projetos, v. 4, n. 2, p. 26-53, São Paulo, 2009.

TESSARO, A. B. et al. Quantificação e classificação dos resíduos procedentes da construção civil e demolição no município de Pelotas, RS. Ambiente Construído, v. 12, n. 2, p. 121-130, Porto Alegre, 2012.

TOLEDO, R. et al. A difusão de inovações tecnológicas na indústria da construção civil. In: Encontro Nacional de Tecnologia do Ambiente Construído, 8., 2000. Anais... Bahia: ANTAC, 2000.

WRAP, Waste \& Resources Action Programme, Guide 2010. Designing out Waste: a design team guide for buildings. Disponível em: < http://www.wrap.org.uk/content/designing-out-waste-design-team-guide-buildings-0>. Acesso em 02 abr. 2015. 